https://helda.helsinki.fi

Swift heavy ion effects on DLC-nanotube-diamond thin films

\author{
Ren, Wei
}

2017-09-06

Ren , W , Djurabekova , F \& Nordlund , K 2017 , ' Swift heavy ion effects on

DLC-nanotube-diamond thin films ' , Journal of Physics. D, Applied Physics , vol. 50 , no. 35

, 355301 . https://doi.org/10.1088/1361-6463/aa7e21

http://hdl.handle.net/10138/308617

https://doi.org/10.1088/1361-6463/aa7e21

acceptedVersion

Downloaded from Helda, University of Helsinki institutional repository.

This is an electronic reprint of the original article.

This reprint may differ from the original in pagination and typographic detail.

Please cite the original version. 


\title{
Swift heavy ion effects on DLC-nanotube-diamond thin films
}

\author{
Wei Ren ${ }^{1, *}$, Flyura Djurabekova ${ }^{1,2}$, Kai Nordlund ${ }^{1}$ \\ ${ }^{1}$ Department of Physics, University of Helsinki, P.O. Box 43, FIN-00014 UNIVERSITY OF HELSINKI, Finland. \\ ${ }^{2}$ Helsinki Institute of Physics, University of Helsinki, P.O. Box 43, FIN-00014 UNIVERSITY OF HELSINKI, Finland. \\ *Email: wei.ren@helsinki.fi
}

\begin{abstract}
The composites based on a mixture of carbon nanotubes and diamond-like-carbon(DLC)-CNT are of great interest due to the potential to achieve unique electronic and optical properties with enhanced mechanical wear resistance. Swift heavy ion (SHI) irradiation of such carbon nanostucture composites suggest a way to tune the material's properties via localized structural modifications. We use classical molecular dynamics simulations combined with an inelastic thermal spike model to study the mechanisms of track formation by SHI in DLC-CNT-diamond thin films. We observe a clear increase of content of atoms with $\mathrm{sp}^{2}$ hybridization compared to the initial structure. When the system reached an equilibrium state after the energy deposition, the track was structurally expanded outwards from the most underdense core. In addition, we found that the track radii are different in different composites, with smaller track radii in pure diamond and larger radii in DLC. Sputtering occurred predominantly from the track center.
\end{abstract}

\section{Introduction}

High energy heavy ion irradiation is known to create cylindrical damage regions in the irradiated materials, known as ion tracks [1]. With the development of large accelerator facilities, which are able to provide welldefined heavy ion irradiation conditions, the track formation has been extensively investigated [1-4]. Ion-induced material modifications and tracks have been characterized by several experimental techniques, for example, atomic-force microscopy (AFM) [2], high resolution transmission electron microscopy(HRFEM) [3], and extended X-ray absorption fine structure(EXAFS) [4].

Diamond-like-Carbon(DLC) is a metastable form of amorphous carbon, which contains a significant fraction of $\mathrm{sp}^{3}$ bonds. As DLC films are well known for their hardness and high elastic modulus, they are utilized in a wide range of applications to improve properties such as high wear resistance, chemical inertness, high electrical resistivity, and low friction coefficients [5].

DLC-CNT composites, where the functional CNTs are covered by the amorphous DLC matrix, are of great interest for nanotechnology applications. If the system is set up in such a way that the irradiation affects only the outer layer of DLC but not the buried CNT layer, one can achieve a condition where the CNT properties of electrical conductivity and optical transparency are preserved, while wear resistance is enhanced [6].

Both experiments and simulations found that swift heavy projectiles in DLC lead to extended defects and structural modifications, with the production of nanohillocks on the surface along the ion trajectory [7]. Previous studies on ion-tracks in DLC show that SHI irradiations create conducting tracks embedded in the originally insulating DLC material, due to the transformation of $\mathrm{sp}^{3}$ to $\mathrm{sp}^{2}$ hybridization in the tracks [8-10].

Since experimental study of the irradiation effects can only investigate the sample state post mortem, the actual material modification processes occurring on a picosec- ond timescale are difficult to determine by measurements only. Atomistic simulations are well suited to examine the evolution of the structural changes up to timescales of tens of ps.

The slowing down of swift heavy ions (SHI) is dominated by inelastic interactions with the target electrons. In this paper, we apply the inelastic thermal spike model (i-TS) $[11,12]$ in classical molecular dynamics (MD) simulations to examine the swift heavy ion irradiation effects on DLC-CNT-diamond thin films.

\section{Simulation methods}

The swift heavy ion impact processes were simulated by using the classical MD method with the PARCAS code[13-16]. For modelling the C-C interactions, the empirical Brenner-Beardmore potential [17], which is an implementation of the original Brenner potential [18-20], was used. The extended cut-off parameters $(\mathrm{R}=1.95$ $\AA, \mathrm{S}=2.25 \AA$ ) were implemented, due to the fact that significant fractions of $\mathrm{sp}^{3}$ hybridization can be achieved only with these adjusted cutoff parameters, as reported by Jäger and Albe[21].

\section{Structures}

The DLC-CNT-diamond thin films were prepared by plasma deposition in our previous work [22], where successive low energetic carbon atoms were irradiated on a diamond substrate. A box size of about $41 \times 36 \times 12$ $\AA^{3}$ was used as the diamond $\{111\}$ substrate with about 6 atomic layers. The multi-walled carbon nanotubes (MWCNTs) were composed of $(5,5),(10,10),(15,15)$ armchair SWCNTs, which gave the diameters of $6.8 \AA, 13.6$ $\AA$, and $20.3 \AA$, with the equilibrium inter-shell distance of $3.4 \AA$. Before the deposition, the CNTs-diamond substrate was built by connecting the MWCNT on the mid- 


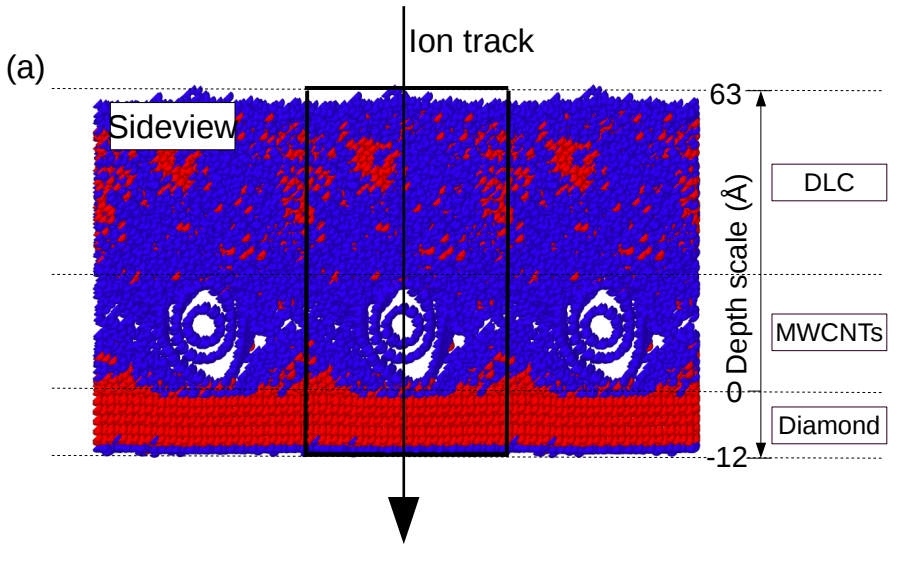

(b)

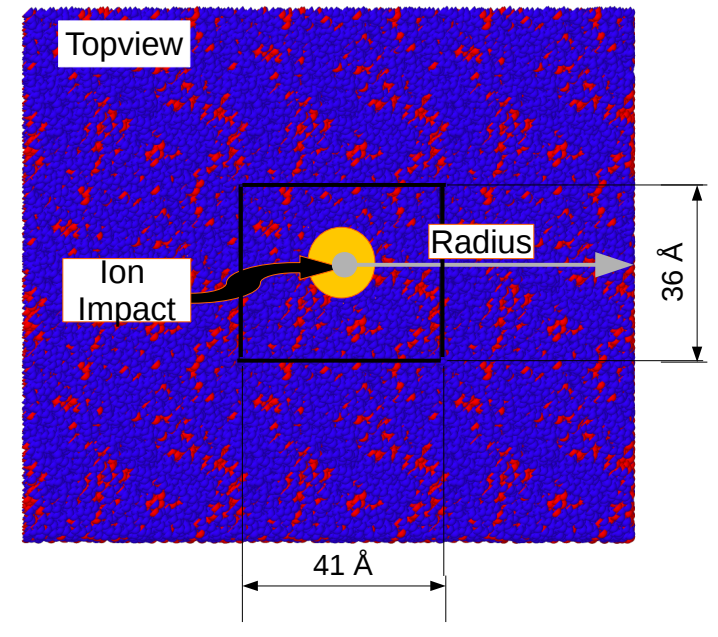

FIG. 1. The visualizations of the relaxed DLC-CNT-diamond thin film (a) sideview and (b) topview. Due to the size of the figure, only the center part of the system with 3 replicated single cells (out of a total of 10) is shown.

dle surface of the diamond cell with the distance of 2.0 $\AA$ in between, followed by a 100 ps relaxation.

In the plasma deposition process, periodic boundary conditions were used at the lateral directions, while the top surface was kept open. Before each bombardment, a random cell shift in the lateral directions over the periodic boundaries was used. The low energy carbon atoms of $30 \mathrm{eV}$ and $50 \mathrm{eV}$ bombarded the open surface perpendicularly at temperatures of $100 \mathrm{~K}$ and $300 \mathrm{~K}$, respectively. The two different temperatures were used to obtain the different $\mathrm{sp}^{3}$ content of the substrate. As we showed earlier [22], the lower ambient temperature creates a higher $\mathrm{sp}^{3}$ hybridization fraction of growing DLC films. For each bombardment within the time scale of $15 \mathrm{ps}$, Berendsen temperature scaling [23] was used to dissipate heat from the impact region to the rest of the system, by which the lateral periodic boundaries within a thickness of $7 \AA$ were immersed in a heat bath. After each bombardment, a relaxation time of 5 ps was used to linearly cool down the entire system.

After a certain amount of bombardments to achieve a sufficient thickness of DLC, the DLC-CNT-diamond single cell was replicated 10 times in the lateral directions to obtain an adequately large film substrate with the size of about $410 \times 360 \times 75 \AA^{3}$ for the swift heavy ion irradiation. Then, the replicated film was relaxed to release the stress in the lateral directions. The cell is illustrated in Fig. 1, where only the central part of the system with 3 replicated single cells is shown..

\section{Ion energy deposition}

The swift heavy ion irradiation was modelled by the inelastic thermal spike model (i-TS) [11, 12]. In this approach, the initial energy deposition (where a certain number of atoms within an initial track radius is given an initial excitation energy), is connected with the subsequent diffusion process of the electronic and lattice subsystems, linked by the electron-phonon coupling [24]. The impact of $1.14 \mathrm{GeV} \mathrm{U}(4.8 \mathrm{MeV} / \mathrm{u})$ swift heavy ion with the stopping power of $35 \mathrm{keV} / \mathrm{nm}$ was modelled by giving the initial excitation energy according to the radial kinetic energy distribution profile calculated using the i-TS, where the electron-phonon coupling constant $10^{13} \mathrm{~J} /\left(\mathrm{sKcm}^{3}\right)$ was deduced from the heat conductivity of graphite [24]. According to the initial energy deposition, in the MD simulations the temperature of the system instantaneously reached the maximum within the time scale of $100 \mathrm{fs}$. The kinetic energy distribution of the entire system was then cooled down by immersing the lateral periodic boundaries with the thickness of $15 \AA$ in the heat bath at $100 \mathrm{~K}$ or $300 \mathrm{~K}$. The SHI process was simulated for $700 \mathrm{ps}$, which was found to be long enough to completely cool down the system to an equilibrium state.

\section{Analysis}

The structural changes were characterized by examining the changes of atomic bonding configurations of $\mathrm{sp}^{2}$ and $\mathrm{sp}^{3}$ hybridizations with time. The three-fold bonded atoms were considered to be $\mathrm{sp}^{2}$ hybridized, while the four-fold bonded atoms were considered to be $\mathrm{sp}^{3}$ hybridized. We analyzed the density variation within the track by calculating its value within concentric cylindrical rings with the width of $10 \AA$ A In addition, bonding topology [25], depth variation of the track radius as well as sputtering yield were analysed to obtain deeper insight of the structural changes. 


\section{(a) $300 \mathrm{~K}$}

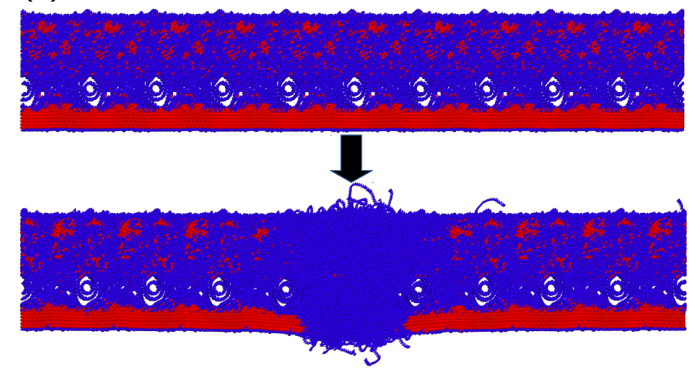

(b) $100 \mathrm{~K}$

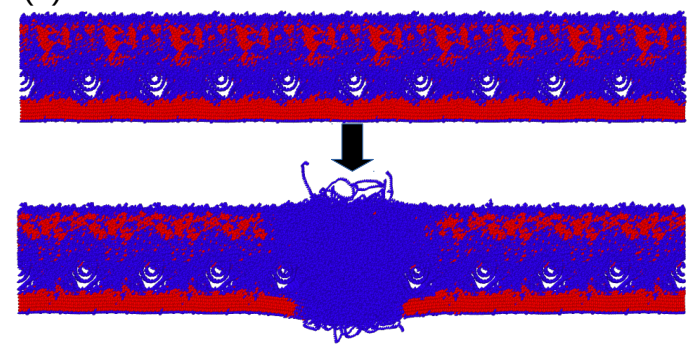

FIG. 2. Cross sections of the equilibrium system after SHI impact (a) at $300 \mathrm{~K}$ and (b) at $100 \mathrm{~K}$. The blue atoms are $\mathrm{sp}^{2}$ bonded, while the red atoms are $\mathrm{sp}^{3}$ bonded.

\section{Results and discussion}

To illustrate the track formation after the SHI impact, the cross sections of the initial frames and the frames at 700 ps are presented in Fig. 2 for both temperatures. In this figure, it is clearly seen that the DLC film grown at $100 \mathrm{~K}$ exhibits the higher content of $\mathrm{sp}^{3}$ hybridization before the SHI impact, compared to the film grown at 300 $\mathrm{K}[22]$. After SHI impact at ambient temperatures of $300 \mathrm{~K}$ and $100 \mathrm{~K}$, respectively, the embedded MWCNTs were completely destroyed in the impact region. Moreover, the obviously predominance of blue atoms ( $\mathrm{sp}^{2}$ hybridization) in this region can be interpreted to be due to the massive conversion of $\mathrm{sp}^{3}$ to $\mathrm{sp}^{2}$ hybridization, which illustrates well the track formation.

\section{Graphitization}

To analyze in detail the mechanisms of track formation during the impact, the radial profiles of the fractions of $\mathrm{sp}^{3}$ hybridization from the track center are calculated at $0,0.1,0.5,1,2,5,700 \mathrm{ps}$ for the cases of $300 \mathrm{~K}$ and $100 \mathrm{~K}$ in Fig. 3(a1) and Fig. 3(b1), respectively. We can see that before the energy deposition, the fraction of $\mathrm{sp}^{3}$ bonded atoms was evenly distributed around 0.32 for $300 \mathrm{~K}$ and 0.4 for $100 \mathrm{~K}$ through the entire cell. At 1 ps, when the temperature in the track core had already reached the maximum after the energy depositions, the $\mathrm{sp}^{3}$ fractions decreased considerably within the tracks, while at the radius far enough from the track cores $(\geq$ $100 \AA)$, the $\mathrm{sp}^{3}$ hybridizations remained intact. In the track cores, the $\mathrm{sp}^{3}$ fractions decreased to 0.17 for the case of irradiation at $300 \mathrm{~K}$ and 0.19 for that at $100 \mathrm{~K}$, nearly half of the values observed in the structures before the energy deposition. This illustrates that nearly half of the $\mathrm{sp}^{3}$ bonded atoms are converted to $\mathrm{sp}^{2}$ bonded atoms already after $1 \mathrm{ps}$. After $2 \mathrm{ps}$, there were no $\mathrm{sp}^{3}$ hybridizations observed within a radius of $30 \AA$ from the core center.

Fig. 3(a2) and Fig. 3(b2) show the density distributions over the radius from the core center before the energy deposition and after $700 \mathrm{ps}$ at $300 \mathrm{~K}$ and $100 \mathrm{~K}$, respectively. Before the SHI impact, the densities of the DLCCNTs-diamond systems were roughly evenly distributed around $2.51 \mathrm{~g} / \mathrm{cm}^{3}$ at $300 \mathrm{~K}$ and $2.61 \mathrm{~g} / \mathrm{cm}^{3}$ at $100 \mathrm{~K}$, falling within the range of the densities of diamond (3.52 $\left.\mathrm{g} / \mathrm{cm}^{3}\right)$ and graphite $\left(2.09-2.27 \mathrm{~g} / \mathrm{cm}^{3}\right)$. When the systems reached the equilibrium states after $700 \mathrm{ps}$ after the energy deposition, we can see the formation of a strongly pronounced underdense core with $1.88 \mathrm{~g} / \mathrm{cm}^{3}$ and 1.91 $\mathrm{g} / \mathrm{cm}^{3}$ at $300 \mathrm{~K}$ and $100 \mathrm{~K}$, respectively. No densification is observed in the shell of the structure, showing that the buried layer of CNTs has been destroyed.

We also plot the depth profiles of the $\mathrm{sp}^{3}$ fraction in the entire system within the depth interval of $2.05 \AA$ at different times after the energy was deposited to the system to analyze the evolution of relative reduction of $\mathrm{sp}^{3}$ fraction in the structure due to the ion track, as shown in Fig. 3(a3) and Fig. 3(b3). Such analysis will exhibit the change of the quality of the DLC structure with depth. The negative values of depth show the grown DLC film, and the positive values show the diamond substrate with the interface at $0 \AA$. Before the SHI (as we have studied in Ref [22]), the atoms with $\mathrm{sp}^{3}$ hybridization were barely observed at the depths between -25 and $0 \AA$, where the curvature of the MWCNT did not allow for four-fold bonding. However, beyond this range, the $\mathrm{sp}^{3}$ hybridizations increased gradually to more stable values, up to $\sim$ $35 \%$ in the cells grown at $300 \mathrm{~K}$ and $\sim 65 \%$ in the cells grown at $100 \mathrm{~K}$. After $700 \mathrm{ps}$, the values of the $\mathrm{sp}^{3}$ content over the depth did not change, only slightly shifted toward the diamond substrate. The relative reduction of $\mathrm{sp}^{3}$ atoms overall in the structure is not significant, which indicates that only a small fraction of atoms within the track core has changed their hybridization.

The primitive ring analysis shown in Fig. 3(a4) and Fig. 3(b4) demonstrates how the total number of primitive rings [25] changes after the irradiation. These numbers are averaged within the concentric cylindrical rings to be shown as radial distributions from the center of the track. Before the energy deposition, the 6-membered rings dominate, indicating the ordered structure. However, after the SHI was introduced, we can see that the number of 6-membered rings per atom decreased dramat- 
(a1)

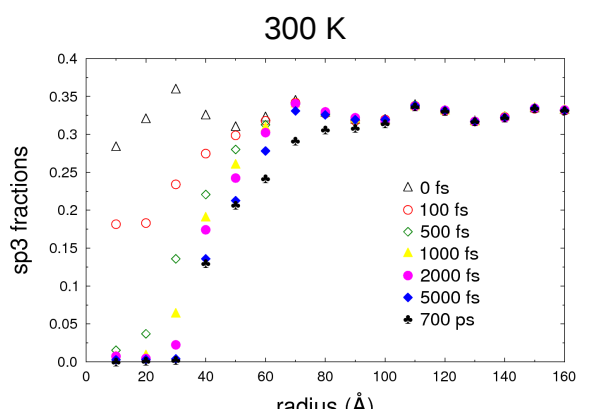

(a2)

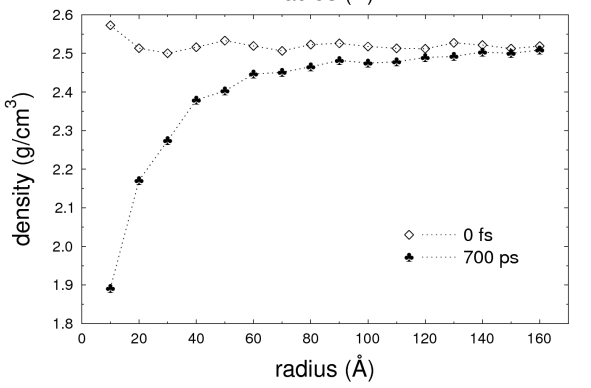

(a3)

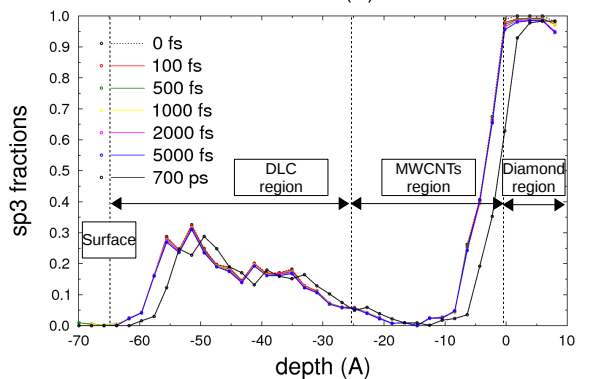

(a4)

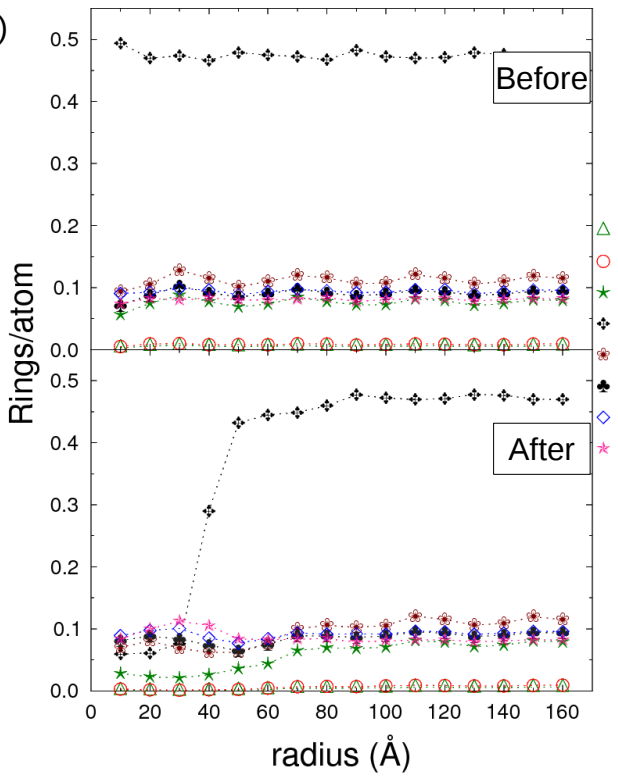

(b1)

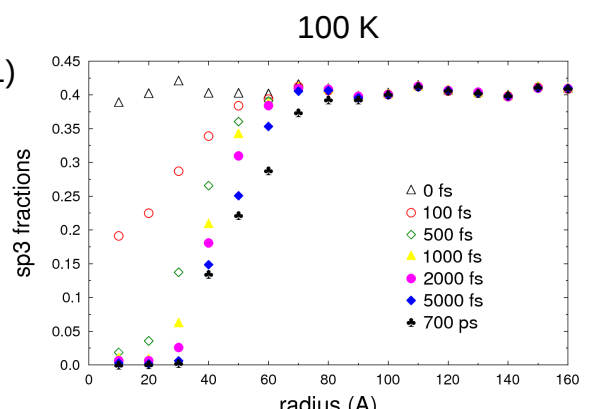

(b2)

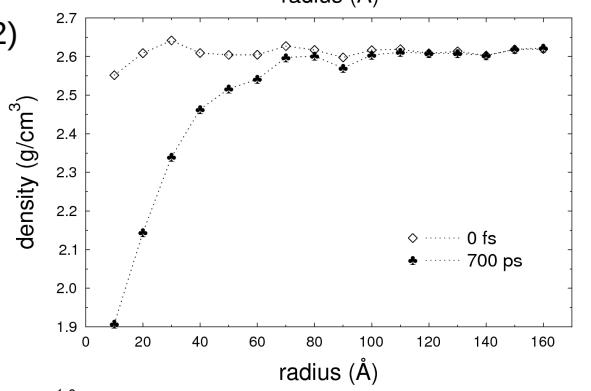

(b3)
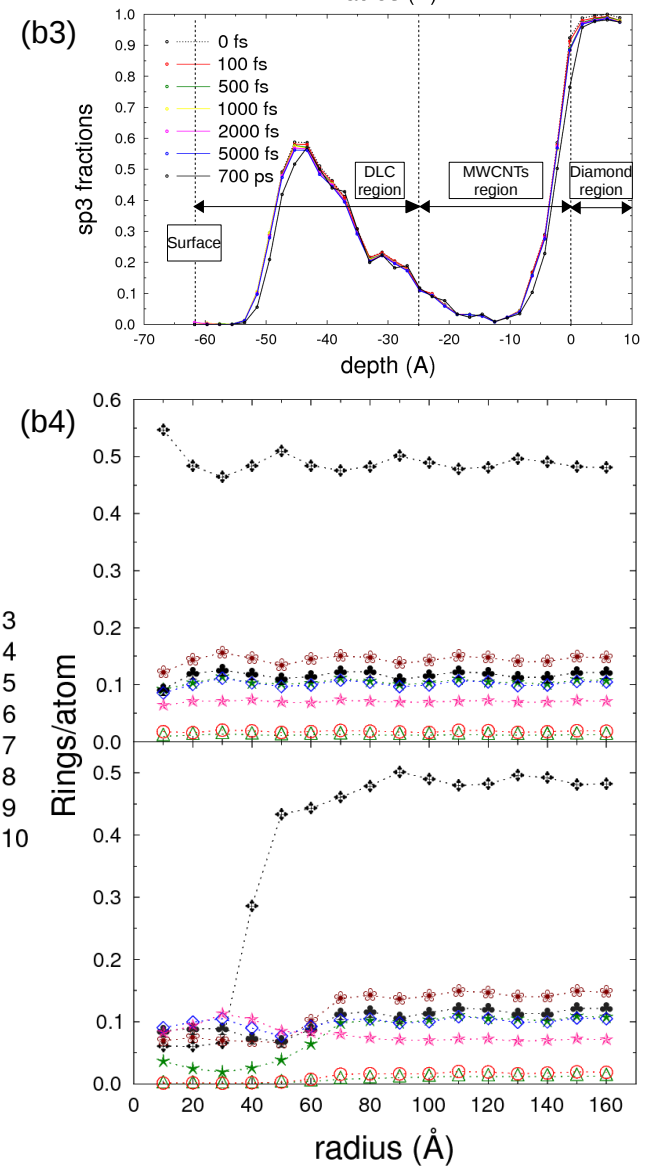

FIG. 3. For the system at $300 \mathrm{~K}$ with DLC deposition energy of $30 \mathrm{eV}$ (left panel), and for the system at $100 \mathrm{~K}$ with DLC deposition energy of $50 \mathrm{eV}$ (right panel). (a/b): 1) radial profiles of $\mathrm{sp}^{3}$ fractions at different time scales during the irradiation. The SHI bombarded perpendicularly the surface at the radius value of $0 ; 2$ ) radial profiles of mass densities before and after the irradiation; 3) depth profiles of $\mathrm{sp}^{3}$ fractions at different time scales; The deposited DLC films are at the negative depth ranges, while the MWCNTs-diamond interface is at $0 \AA$. 4) radial profiles of rings per atom for different ring sizes, before and after the SHI impact. 


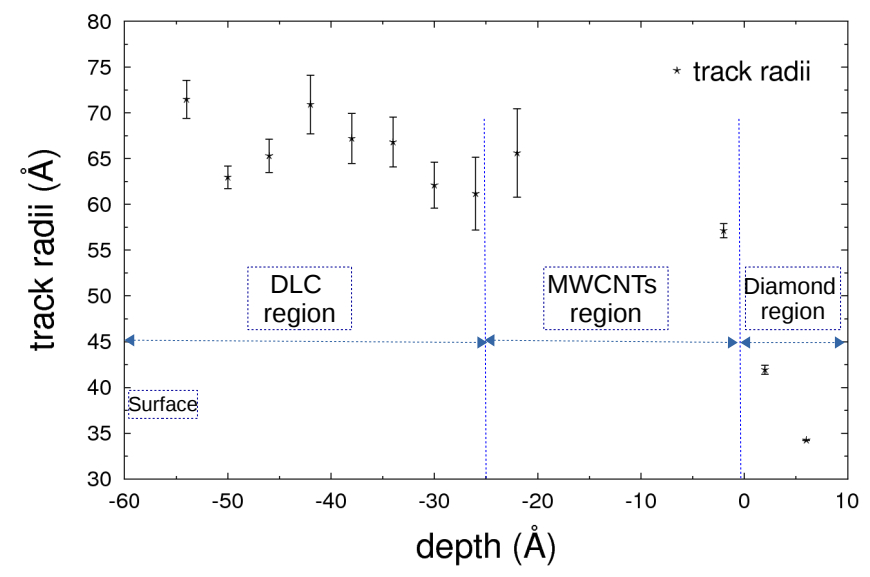

FIG. 4. The depth profile of track radii for the system at 300 $\mathrm{K}$ with DLC deposition energy of $30 \mathrm{eV}$.

ically within the first cylinder of radius $30 \AA$, becoming much closer to the number of 7-, 8-, 9-, 10-membered rings, showing a completely disordered structure. Meanwhile, the number of 5- and 7-membered rings notably decreased, while 9- and 10-membered rings somewhat increased in the center region of the track. As we saw in Ref [22], a structure with higher $\mathrm{sp}^{3}$ fraction has a higher fraction of 6-membered rings. Thus, the ring analysis further supports the conclusion that the track formed by a SHI is prone to graphitization, as extra free volume is created during thermal expansion after the impact.

\section{Variation of track radius over the depth}

Since the cell which we used in the current simulations was highly inhomogeneous, we analyzed how the radius of the track varied with the depth. For this, we analyzed the depth profile of $\Delta \mathrm{sp}^{3}$ (the change of $\mathrm{sp}^{3}$ fractions before and after SHI) for the structure obtained at 300 $\mathrm{K}$. The calculated values of $\Delta \mathrm{sp}^{3}$ were then fitted to a Fermi function with the form:

$$
f=\frac{A_{0}}{\left(e^{\frac{r-r_{0}}{w}}+1\right)}+C
$$

where $A_{0}$ is the amplitude of the $\Delta \mathrm{sp}^{3}$ distribution, $r_{0}$ is the track radius, and $w$ is the width of the transition region. Fig. 4 shows the distribution of the fitted track radii along the $\mathrm{z}$ coordinate (depth profile). We can see that in the diamond substrate region at the depth between 0 and $10 \AA$, the track radius is around $34 \AA$. In the DLC region, at the depth between -55 and -20 $\AA$, the track became significantly larger, with the radius values approximately 60 - $70 \AA$. At the interface between the CNTs and the diamond, at the depth around $0 \AA$, there was a certain amount of $\mathrm{sp}^{3}$ hybridization growing near the surface of diamond, which explains well why

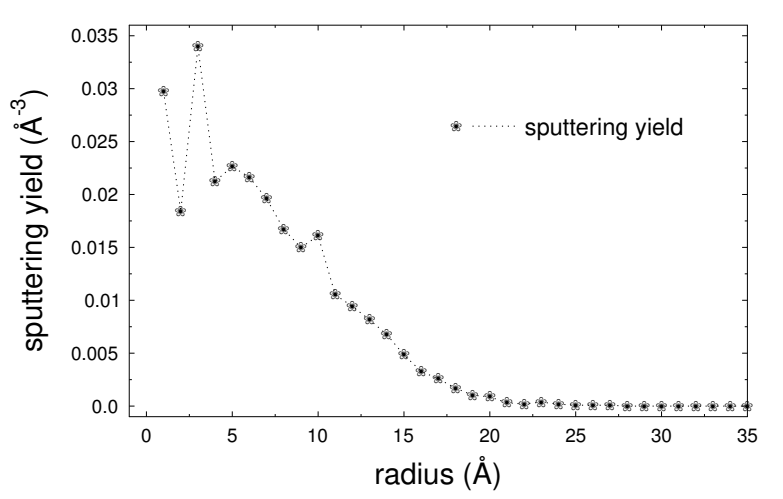

FIG. 5. The radial profile of sputtering yield for the system at $300 \mathrm{~K}$ with DLC deposition energy of $30 \mathrm{eV}$.

the track radius at this region increased from $35 \AA$ to 55 $\AA$. In our current work, the stopping power of the SHI in the system is $35 \mathrm{keV} / \mathrm{nm}$, which is much larger than the threshold stopping power of $19 \mathrm{keV} / \mathrm{nm}$ of track formation in diamond as studied in Ref [26]. Hence, we can say that the size of the track radius induced by SHI impact depends highly on the structure of the carbon, where the SHI impact creates a much larger track in DLC compared to diamond.

\section{Sputtering yield}

Fig. 5 shows the radial distribution of sputtering yield from the core center for the system grown at $300 \mathrm{~K}$. The values of the sputtering yield are normalized by the radial concentric cylindrical volumes. It shows that the number of sputtered atoms near the core center within the radius of $5 \AA$ is relatively higher, while it gradually decreased to 0 at the radius of $30 \AA$. There are no sputtered atoms outside of the $30 \AA$ radius. Hence, we can say that the sputtering takes place only at the very center of the track core and does not play a significant role on the formation of the track structure.

\section{Conclusions}

We have used MD simulations to study the SHI impact on composite DLC-CNT-diamond thin films. We found that there were no $\mathrm{sp}^{3}$ hybridization observed near the track core, due to the conversion of $\mathrm{sp}^{3}$ bondings to $\mathrm{sp}^{2}$ bondings. The structure of the track was expanded progressively from the core center with the most underdense densities of $1.88 \mathrm{~g} / \mathrm{cm}^{3}$ at $300 \mathrm{~K}$ and $1.91 \mathrm{~g} / \mathrm{cm}^{3}$ at 100 $\mathrm{K}$. The SHI impact induced a completely disordered ring topology, which confirms the graphitization during the track formation. 


\section{Acknowledgements}

The sponsorship of the Academy of Finland through research project HISCON and MESIOS (Project No.220911 and 259595) is acknowledged. Grants of com- puter time from the Center for Scientific Computing in Espoo, Finland are gratefully acknowledged.

\section{References}

[1] R. Fleischer, P. Price, and R. Walker, Journal of applied Physics 36, 3645 (1965).

[2] F. Thibaudau, J. Cousty, E. Balanzat, and S. Bouffard, Physical review letters 67, 1582 (1991).

[3] C. Houpert, F. Studer, D. Groult, and M. Toulemonde, Nuclear Instruments and Methods in Physics Research Section B: Beam Interactions with Materials and Atoms 39, 720 (1989).

[4] F. Studer, C. Houpert, M. Toulemonde, and E. Dartyge, Journal of solid state chemistry 91, 238 (1991).

[5] A. Grill, Diamond and related materials 8, 428 (1999).

[6] A. Kaskela, J. Koskinen, H. Jiang, Y. Tian, X. Liu, T. Susi, M. Kaukonen, A. G. Nasibulin, and E. I. Kauppinen, Carbon 53, 50 (2013).

[7] D. Schwen, E. Bringa, J. Krauser, A. Weidinger, C. Trautmann, and H. Hofsäss, Applied Physics Letters 101, 113115 (2012).

[8] M. Waiblinger, C. Sommerhalter, B. Pietzak, J. Krauser, B. Mertesacker, M. C. Lux-Steiner, S. Klaumünzer, A. Weidinger, C. Ronning, and H. Hofsäß, Applied Physics A 69, 239 (1999).

[9] J.-H. Zollondz, D. Schwen, A.-K. Nix, C. Trautmann, J. Berthold, J. Krauser, and H. Hofsäss, Materials Science and Engineering: C 26, 1171 (2006).

[10] J. Krauser, J.-H. Zollondz, A. Weidinger, and C. Trautmann, Journal of applied physics 94, 1959 (2003).

[11] Z. Wang, C. Dufour, E. Paumier, and M. Toulemonde, Journal of Physics: Condensed Matter 6, 6733 (1994).

[12] P. Kluth, C. Schnohr, O. Pakarinen, F. Djurabekova, D. Sprouster, R. Giulian, M. C. Ridgway, A. Byrne, C. Trautmann, D. Cookson, et al., Physical review letters 101, 175503 (2008).

[13] K. Nordlund, (2006), PARCAS computer code. The main principles of the molecular dynamics algorithms are pre- sented in $[14,15]$. The adaptive time step and electronic stopping algorithms are the same as in [16].

[14] K. Nordlund, M. Ghaly, R. Averback, M. Caturla, T. D. de La Rubia, and J. Tarus, Physical Review B 57, 7556 (1998).

[15] M. Ghaly, K. Nordlund, and R. Averback, Philosophical Magazine A 79, 795 (1999).

[16] K. Nordlund, Computational materials science 3, 448 (1995).

[17] K. Beardmore and R. Smith, Philosophical Magazine A 74, 1439 (1996).

[18] D. W. Brenner, Physical Review B 42, 9458 (1990).

[19] D. W. Brenner, Physical Review B 46, 1948 (1992).

[20] J. Tersoff, D. Vanderbilt, and V. Vitek, Atomic scale calculations in materials science, Tech. Rep. (Pittsburgh, PA (USA); Materials Research Society, 1989).

[21] H. Jäger and K. Albe, Journal of applied physics 88, 1129 (2000).

[22] W. Ren, A. Iyer, J. Koskinen, A. Kaskela, E. I. Kauppinen, K. Avchaciov, and K. Nordlund, Journal of Applied Physics 118, 194306 (2015).

[23] H. J. Berendsen, J. v. Postma, W. F. van Gunsteren, A. DiNola, and J. Haak, The Journal of chemical physics 81, 3684 (1984).

[24] M. Toulemonde, W. Assmann, C. Dufour, A. Meftah, and C. Trautmann, Nuclear Instruments and Methods in Physics Research Section B: Beam Interactions with Materials and Atoms 277, 28 (2012).

[25] X. Yuan and A. Cormack, Computational materials science 24, 343 (2002).

[26] O. H. Pakarinen, F. Djurabekova, and K. Nordlund, Nuclear Instruments and Methods in Physics Research Section B: Beam Interactions with Materials and Atoms 268, 3163 (2010). 